\title{
Direct Writing and Actuation of Three- Dimensionally Patterned Hydrogel Pads on Micropillar Supports
}

\section{Citation}

Zarzar, Lauren D., Philseok Kim, Mathias Kolle, C. Jeffrey Brinker, Joanna Aizenberg, and Bryan Kaehr. 2011. "Direct Writing and Actuation of Three-Dimensionally Patterned Hydrogel Pads on Micropillar Supports." Angew. Chem. Int. Ed. 50, no. 40: 9356-9360. doi:10.1002/anie.201102975.

\section{Published Version}

doi:10.1002/anie.201102975

\section{Permanent link}

http://nrs.harvard.edu/urn-3:HUL.InstRepos:34611664

\section{Terms of Use}

This article was downloaded from Harvard University's DASH repository, and is made available under the terms and conditions applicable to Open Access Policy Articles, as set forth at http:// nrs.harvard.edu/urn-3:HUL.InstRepos:dash.current.terms-of-use\#OAP

\section{Share Your Story}

The Harvard community has made this article openly available.

Please share how this access benefits you. Submit a story.

\section{Accessibility}




\title{
Direct Writing and Actuation of 3D-Patterned Hydrogel Pads on Micropillar Supports
}

\author{
Lauren D. Zarzar, Philseok Kim, Mathias Kolle, C. Jeffrey Brinker, Joanna Aizenberg,* and Bryan \\ Kaehr*
}

Many biological organisms employ micro- and nanoscale systems to actuate structural components with a high degree of spatial control. The resulting patterned or predetermined movement of the components gives rise to versatile biological materials with locally reconfigurable features and region-specific dynamic properties. On the molecular level, biological systems may regulate the availability of catalytic sites on enzymes by local reconfiguration of the protein structure, such as in allosteric modulation. ${ }^{[1]}$ On the microscale, echinoderms use actuating pedicellariae for particle capture and release and body cleaning, ${ }^{[2]}$ and bacteria employ the movement of flagella to generate directional locomotion. ${ }^{[3]}$ Squid use the mechanical expansion and contraction of chromatophores to reversibly change color and pattern for camouflage and communication. ${ }^{[4]}$ These systems provide inspiration for the development of artificial 'smart' materials and surfaces with similar properties that respond autonomously and reversibly to

[*] Prof. C. J. Brinker, Dr. B. Kaehr Advanced Materials Laboratory Sandia National Laboratories 1001 University Blvd. SE, Albuquerque, NM, 87106 (USA) E-mail: bjkaehr@sandia.gov

Department of Chemical and Nuclear Engineering and the Center for Micro-engineered Materials University of New Mexico Albuquerque, NM, 87106 (USA)

L. D. Zarzar, Dr. P. Kim, Dr. M. Kolle, Prof. J. Aizenberg Department of Chemistry and Chemical Biology, School of Engineering and Applied Sciences, and the Wyss Institute for Biologically Inspired Engineering Harvard University 29 Oxford Street, Cambridge, MA 02138 (USA) Email: jaiz@seas.harvard.edu

[**] We thank Dr. M. Aizenberg for discussions. This work was supported by the National Institute for Nano Engineering (NINE) program at Sandia National Laboratories; the Air Force Office of Scientific Research grant 9550-10-1-0054; and U.S. Department of Energy, Office of Basic Energy Sciences, grant DE-SC0005247. Sandia is a multiprogram laboratory operated by Sandia Corporation, a Lockheed Martin Company, for the United States DOE's NNSA under contract DE-AC04-94AL85000. B.K. gratefully acknowledges the Sandia National Laboratories Truman Fellowship in National Security Science and Engineering and the Laboratory Directed Research and Development program for support. L. D. Z thanks the Department of Defense for support through the National Defense Science and Engineering Graduate Fellowship Program, as well as the National Science Foundation for support through the Graduate Research Fellowship Program.

Supporting information for this article is available on the WWW under http://www.angewandte.org or from the author. environmental cues. Recently such reversibly responsive materials, particularly those patterned or manipulated on the nano- and microscale, have been the subject of intense research ${ }^{[5]}$ due to their promising impact in areas including sensors ${ }^{[6]}$ and actuators, ${ }^{[7]}$ microfluidic systems, ${ }^{[8]}$ microelectromechanical systems, ${ }^{[9]}$ and switchable surfaces with adaptive wettability, optical, mechanical or adhesive properties. $^{[5]}$ In particular, hydrogels can be tailored to respond volumetrically to a wide variety of stimuli including temperature, ${ }^{[10]} \mathrm{pH}^{[11]}$ light, ${ }^{[12]}$ and biomolecules (e.g. glucose) ${ }^{[13]}$, and there has been a significant amount of research and applications devised for this class of materials in areas ranging from tissue engineering ${ }^{[14]}$ to responsive photonics. ${ }^{[15]}$

We recently described a responsive and reversibly actuating surface based on a hybrid architecture consisting of passive polymeric structural ('skeletal') elements embedded in and under the control of a responsive hydrogel layer ('muscle') attached to a solid support. ${ }^{[16,17]}$ While the volume change of the polymer muscle enables large-area, directional movement of skeletal elements, hydrogel anchoring to a solid support imposes a serious constraint on its capacity for expansion/contraction, and thus limiting the extent of induced actuation of the structural elements. Moreover, this approach does not allow the formation of hydrogel islands that would induce localized actuation of selected areas and the associated regional changes in surface properties.

To expand the opportunities for integration of hydrogels in such composite systems, it would be advantageous to tailor not only the chemistry and swelling properties of the hydrogels but also the size, shape, and placement of the gel in relation to other system components. For example, well-defined, three-dimensionally (3D)patterned, responsive hydrogel pads placed at the tips of micropillars with microscale control would enable nearly unrestricted gel swelling, both in and out of plane, which would actuate the pillars locally with more precise control over the movement of individual elements. While extensive research has been devoted to tailoring the swelling, chemical properties, and responsive behavior of hydrogels, less attention has been paid to the development of patterning protocols that would offer area-specific synthesis and 3D control over micro/nanoscale features of the gel. Many routes to defining hydrogel patterns have been explored including photolithography, ${ }^{[18]}$ soft lithography, ${ }^{[19]}$ and masking techniques ${ }^{[20]}$ but these $2 \mathrm{D}$ approaches lack true control over gel features in 3D, limiting their application for localized microactuation.

Multiphoton lithography (MPL) has emerged as a prominent method for the fabrication of intricately 3D-structured materials with nanoscale precision. ${ }^{[21]}$ Pulsed laser light is focused into a photosensitive reagent solution (e.g., photoresist) to initiate photochemical reactions by a multiphoton absorption process. This nonlinear excitation is restricted to regions of high photon density (i.e. proximal to the focal volume of a focused laser beam), enabling photochemical reactions, such as photopolymerization, to be confined to highly resolved $3 \mathrm{D}$ volumes on the order of $\sim 1 \mathrm{fl}$. MPL has been commonly used with epoxies ${ }^{[21]}$ and acrylic resins ${ }^{[21,22]}$ that 
tend to be rigid when cured, allowing for creation of detailed and stable structures. However, much less attention has been given to MPL of soft materials such as hydrogels. While MPL has been used to polymerize acrylate-based ${ }^{[23-26]}$ and protein-based hydrogels ${ }^{[27]}$, the stimuli-responsive behavior of these hydrogels has not been investigated and only rarely were the swelling properties reported. ${ }^{[25-27]}$

Here, we develop an approach to direct-write reversibly swelling $\mathrm{pH}$ - and temperature-responsive hydrogel patterns onto polymeric micropillars using MPL and examine their localized actuation. For this work, we chose two common and widely studied polymers: temperature-responsive poly( $N$-isopropylacrylamide) (PNIPAAm) and pH-responsive poly(acrylic acid-co-acrylamide) (poly(AAc-Co-AAm)). In a typical experiment, polymer precursors were dissolved in ethylene glycol $(40 \% \mathrm{wt} / \mathrm{wt})$ in the presence of a UV-curable photoinitiator (1\% wt/wt), such as bis(2,4,6trimethylbenzoyl)-phenylphosphine oxide (Irgacure 819), which shows significant absorption around $400 \mathrm{~nm}$ to enable two-photon excitation using 750-800 nm pulsed laser light operating at relatively low average powers. The ethylene glycol was exchanged with water after fabrication. As shown in Figure 1a, hydrogel precursor solution is placed on a micropillar surface and the laser is passed through an objective lens and into the solution; polymerization occurs proximal to the laser focus as it is scanned across the sample (Figure 1b) and hydrogel structures of userdefined shapes ${ }^{[28]}$ can be written directly on the bristles at arbitrary distances above the basal surface (Figure 1c; see Supporting Information for experimental details, and Supporting Movie 1).

Figure 2 shows optical images of a heart-shaped PNIPAAm gel, fabricated at the micropillar tips, which we observed to have a phase transition at $\sim 30^{\circ} \mathrm{C}$, in the contracted $\left(\mathrm{T}>30^{\circ} \mathrm{C}\right)$ and swollen $\left(\mathrm{T}<30^{\circ} \mathrm{C}\right)$ state (Figure $\left.2 \mathrm{~b}\right)$. Poly(AAc-Co-AAm) hydrogels, which are $\mathrm{pH}$ responsive and exhibit a volume phase transition near the $\mathrm{pK}_{\mathrm{a}}$ of acrylic acid (4.25), ${ }^{[29]}$ were attached to the flexible micropillars and fabricated using the same procedure (Figure 2c). These hydrogels (both temperature- and pH-responsive) could be swollen and contracted rapidly (seconds; see Supporting Movie 2)

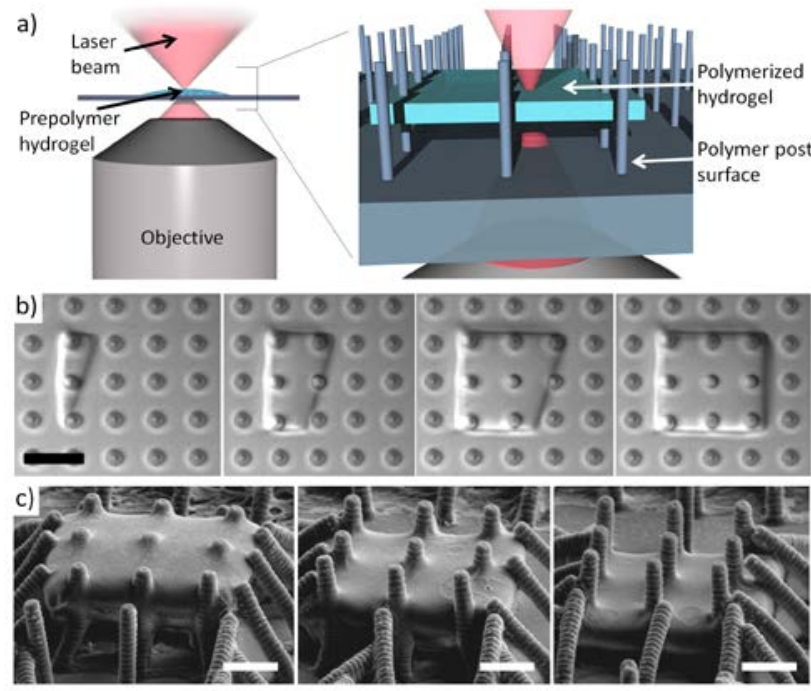

Figure 1. a) Schematic showing the experimental set-up for localized synthesis of MPL hydrogels onto micropillars (height $=10$ $\mu \mathrm{m}$, pitch $=8 \mu \mathrm{m}$, diameter $=1.5 \mu \mathrm{m})$. b) Image sequence of multiphoton-induced polymerization to form a thin hydrogel structure attached to the pillars. (See Supporting Movie 1). Scale bar, $10 \mu \mathrm{m}$. c) SEM images show fabricated hydrogel structures placed precisely along the top, middle, and bottom of the posts. The thickness of the hydrogel pads is $\sim 2 \mu \mathrm{m}$ made by two passes of the scanning laser beam. Scale bars, $5 \mu \mathrm{m}$. a)

b)

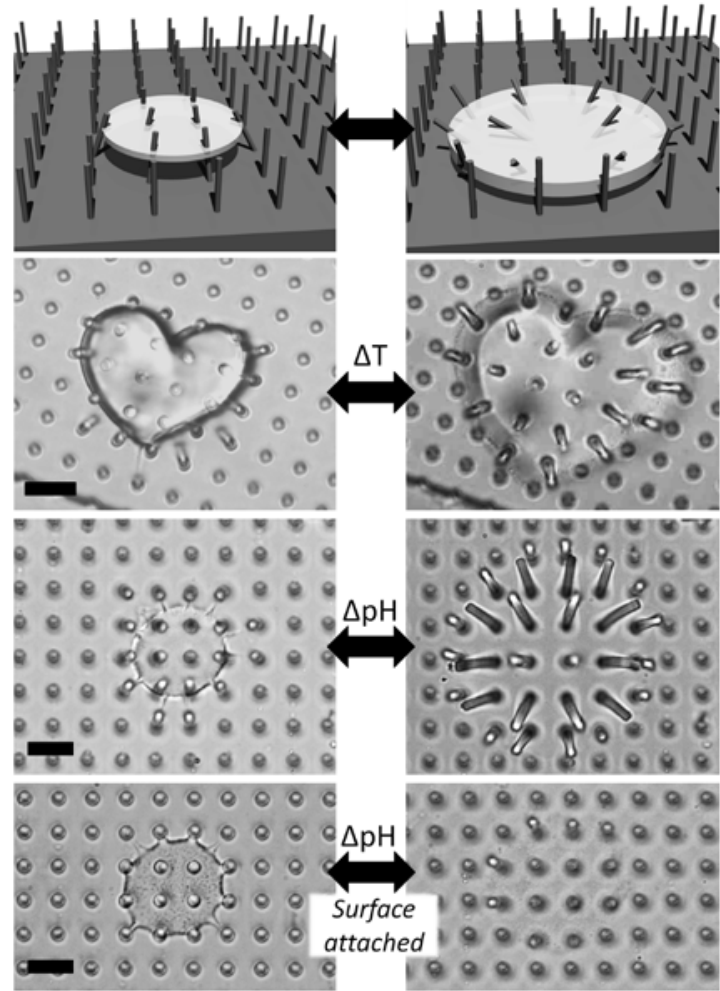

Figure 2. a) Schematic showing the deflection of the flexible pillars via tip-attached hydrogel swelling. Optical microscope images of the contracted and swollen states of b) temperatureresponsive hydrogel (at $\mathrm{T}>30^{\circ} \mathrm{C}$ and $\mathrm{T}<30^{\circ} \mathrm{C}$ ) and c) $\mathrm{pH}$ responsive hydrogel (at $\mathrm{pH}<4.25$ and $\mathrm{pH}>4.25$ ) are shown. $\mathrm{d}$ ) $\mathrm{pH}$ responsive gel under same conditions as (c) but fabricated at the base of the pillars and attached to the basal surface. Scale bars, $10 \mu \mathrm{m}$.

for dozens or more cycles with little deformation in gel structure. Importantly, suspending the hydrogel on the flexible pillars above the substrate surface allows the gel to swell and contract significantly without being constrained by surface attachment. For the thin suspended hydrogel pads, absolute expansion and contraction is greatest along the axes parallel to the surface plane, thus actuating the flexible pillars outward or inward (Figure 2b,c). To demonstrate the importance of suspending the gel, we fabricated a pH-responsive gel attached to the basal surface (Figure 2d) but otherwise identical to the gel shown in Figure 2c. The degree of lateral swelling in response to $\mathrm{pH}$ is significantly constrained due to the surface attachment, and the actuation of the pillars is negligible.

We envision using these 3D-patterned hydrogels as muscle components to actuate artificial filamentous surfaces (or other flexible structures) with a high level of control. To guide these efforts, we investigated how the swelling of the disk-shaped MPLpatterned poly(AAc-co-AAm) hydrogel swelling influences the bending of the epoxy pillars across a range of $\mathrm{pH}$ values between 2 and 8.8 (Figure 3a). Bending angles for three posts (chosen in a single row across the diameter of the disk) exhibit a sharp transition between $\mathrm{pH}=4-5$ in correlation with the expected $\mathrm{pH}$ range for the volume-phase transition. However, the absolute bending angle in either the swollen or contracted state ultimately depends on the position of each post; for posts located near the outer edge of the hydrogel, bending angles between $-20^{\circ}$ and $>70^{\circ}$ were accessible, but for posts near the center of the hydrogel disk the range was much smaller. Assuming that the primary force exerted upon the posts arises from an outwardly-expanding hydrogel located near the tips, the forces exerted upon the microposts were approximated using: 

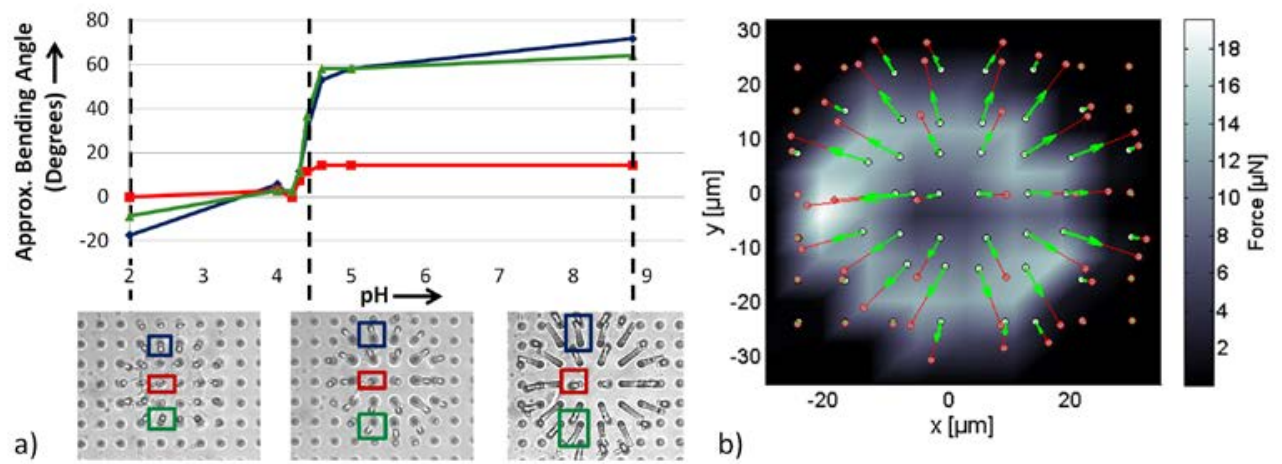

Figure 3. a) Plot of approximate bending angles of pillars that support a pH-responsive hydrogel pad as a function of $\mathrm{pH}$, where a negative angle represents the pillar bending inward toward the center of the gel and a positive angle represents outwardly bending pillars. Posts near the center of the structure (red) show little change in bending. Posts near the edge of the hydrogel (blue and green) bend to very large angles $\left(>70^{\circ}\right)$. b) The displacement of the posts was used to generate a force map of the gel. White circles indicate the initial positions of the tips of the pillars when the gel is contracted, and red circles indicate the position of the tips of the pillars when the gel is swollen. Red lines connect the initial and final positions. Green arrows symbolize the amount of force normalized to the largest force experienced by a pillar in the system. The background color map visualizes the extent of the net force exerted on the pillars in different areas of the gel.

$$
\text { Force }=\frac{3 \pi E r^{4}}{4 h^{3}} * D
$$

where $E$ is the Young's modulus of the glycidyl methacrylatemodified epoxy $(1.5 \mathrm{GPa}){ }^{[16]} r$ is the radius of the posts $(0.75 \mu \mathrm{m})$, $h$ is the height of the posts $(10 \mu \mathrm{m})$, and $D$ is the distance to which the tip of each post is deflected from its initial position. The forces mapped over the surface plane of the disk in Figure 3b indicate that forces on the order of $\mu \mathrm{N}$ were generated near the edge of the swelled gel-sufficient for microscale manipulations where viscous forces on the order of $\mathrm{pN}$ must be overcome. ${ }^{[30]}$ The ability to apply directed forces and dictate filament trajectory via its relative position within the gel provides a foundation to begin to explore actuation schemes over larger distances, for instance, to direct or trap particles with environmental cues using a cilia-like mechanism.

The ability to synthesize interacting hydrogels that respond to different stimuli should prove enabling to realize more complex behaviors not achievable with uniform hydrogel muscles. As shown in Figure 4, the pH-responsive hydrogel and temperature-responsive hydrogel were patterned sequentially as interlocking puzzle pieces. Each hydrogel could be stimulated in an independent manner by

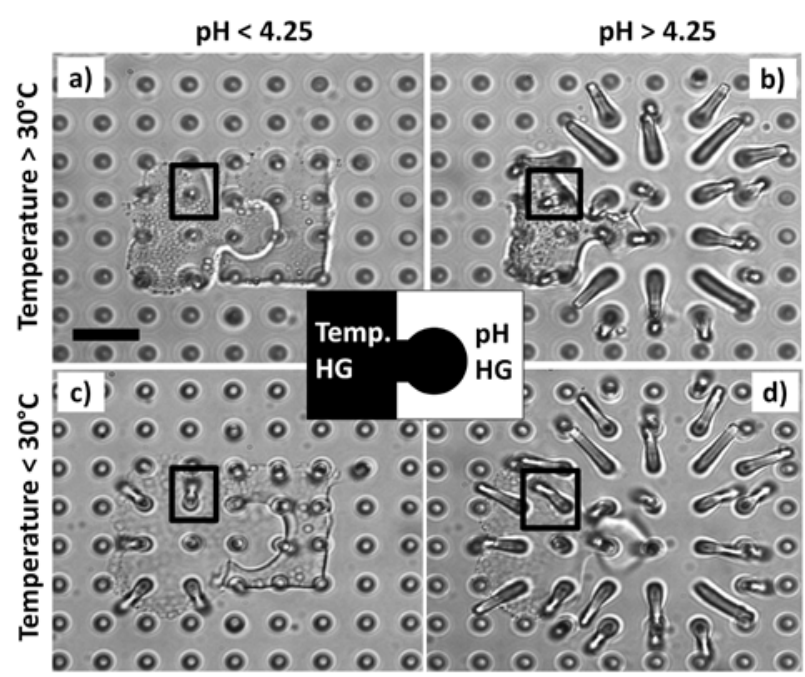

Figure 4. A schematic image showing temperature- and $\mathrm{pH}-$ responsive hydrogels fabricated in close proximity as interlocking puzzle piece shapes (center). Optical microscope images at each temperature and $\mathrm{pH}$ combination are shown (a-d). The black outline highlights an exemplary post as it is bent at four different angles and directions depending on the combination of conditions. Scale bar, 10 $\mu \mathrm{m}$. changing the combination of $\mathrm{pH}$ and temperature. Pillars that had a temperatureresponsive hydrogel on one side and $\mathrm{pH}$-responsive hydrogel on the other, near the interface of the gels, could therefore be actuated to four stable configurations; one such post is highlighted in Figure 4. The shape of the hydrogel at the interface (the interlocking part of the pieces) could be compressed or expanded by the response of the neighboring gel. In Figure 4, for example, comparison of (a) to (b) and (c) to (d) illustrates that at constant temperatures but varying $\mathrm{pH}$, the extruding part of the temperature-responsive hydrogel piece is compressed beyond its equilibrium state by an expanding $\mathrm{pH}$-responsive hydrogel. Similarly, upon comparison of (a) to (c) and (b) to (d) we see that the shape of the $\mathrm{pH}$-responsive hydrogel at the interlocking segment is expanded at constant $\mathrm{pH}$ but varying temperature. The ability to precisely pattern interacting hydrogels of varying responsivity may provide opportunities for the design of systems exhibiting predictive and programmable responses for different combinations of environmental conditions; reconfiguration of structures may now have more than a single "muscle" that controls actuation and induces complex movement of structural elements.

A potent feature of responsive hydrogels is the ability to dynamically adjust and tune the functional density of any chemical moiety in the gel via the volume phase transition. To demonstrate this capability in MPL-generated gels, we co-polymerized a fluorescent polymerizable rhodamine monomer, methacryloxyethyl thiocarbamoyl rhodamine $\mathrm{B}$, with the $\mathrm{pH}$-responsive hydrogel and patterned the gel on the micropillars (Figure 5). We used poly(AAcco-AAm) because of its large swelling response and rhodamine $\mathrm{B}$

a)

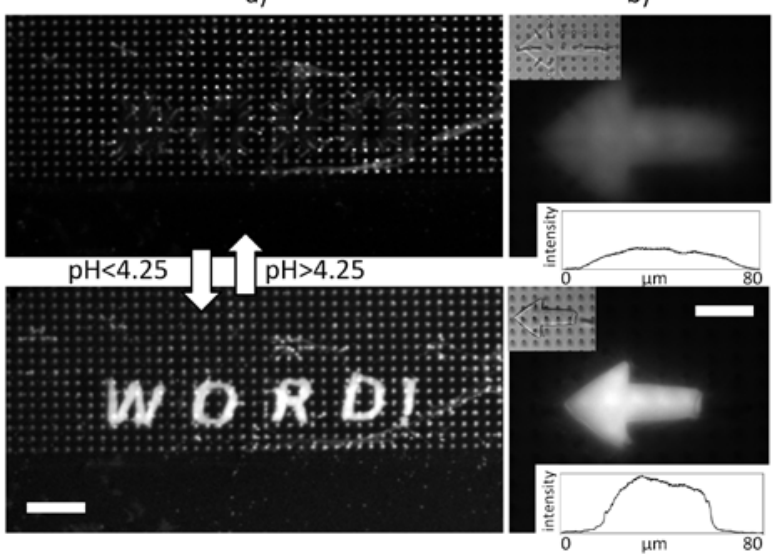

Figure 5. Fluorescence microscope images demonstrating fast and reversible appearance and disappearance of messages due to the change in concentration density of a rhodamine dye which was incorporated into a pH-responsive hydrogel. a) Contraction of the hydrogel in acid increases the fluorescence signal to revea the message "word!". (See Supporting Movie 3). Scale bar, 40 $\mu \mathrm{m}$. b) Contraction of the hydrogel in acid reveals the arrow symbol. Upper insets show DIC images of the gel. Lower insets show the fluorescence intensity profile along the length of the arrow normalized to the bit depth of the fluorescence image. Scale bar, $20 \mu \mathrm{m}$. 
because its fluorescence is insensitive to changes in $\mathrm{pH}$ over the range needed for the swelling response. Expansion and contraction of the fluorophore-modified hydrogel conferred substantial changes in the fluorescence intensity. In particular, suspending the gel above the substrate surface through attachment along the tips of the pillars (as is shown in Figure 1c, left) provided excellent "on/off” behavior of the signal (see Supplementary Movie 3). The significant changes in fluorescence intensity were attributed to the extreme volumephase transition of the unconstrained hydrogel and its influence on the effective density of the fluorophore within the gel volume. Using multiphoton excitation (MPE) point measurements, which provide a means to probe a fixed volume of the hydrogel in both its expanded and contracted states, we measured a $~ 20$-fold increase in the density of rhodamine molecules within gels similar to those pictured in Figure 5. As a result, the message in Figure 5a is clearly seen at $\mathrm{pH}<4.25$ and is effectively "erased" at $\mathrm{pH}>4.25$. Similarly, additional functional components including nanoparticles, ${ }^{[31]}$ peptides, ${ }^{[32]}$ and DNA, ${ }^{[33]}$ can be readily incorporated into these gels and should expand the opportunities for sensors and catalytic systems ${ }^{[34]}$ in which the density and availability of the active sites is tuned via gel response.

In conclusion, we have described a synthetic route to fabricate arbitrary 3D shapes of temperature- and $\mathrm{pH}$-responsive hydrogels using MPL. This procedure allows hydrogels to be precisely positioned in a suspended state on high-aspect-ratio micropillar structures giving rise to significant gel expansion without the surface constraint that typically results from traditional patterning methods in which the gel is attached to the basal surface. The bending angle and actuation direction of microposts are shown to depend on their relative lateral positions (i.e., center versus edge) within the swelling gel, providing a means to direct the movement of post structures with a high degree of tunability. We have shown that this approach makes it possible to pattern interlocking, interacting hydrogels responding to different stimuli and thus to develop unique, complex gel and structural interactions via orthogonal environmental cues. Additionally, hydrogels can be further functionalized, for instance, by introducing a polymerizable fluorophore to generate an optical readout based on the actuationinduced concentration change. MPL-patterned responsive hydrogels incorporating chemical, optical, and catalytic ${ }^{[34]}$ or enzymatic ${ }^{[35]}$ functions will provide great opportunities to develop multicomponent chemical reaction, sensing, and diagnostic systems. Although here we describe micro-patterned 3D 'muscle-like' materials integrated with relatively simple micropillar components, similar 3D microactuation is achievable for more complex 'skeletal' structures that can be fabricated using common MPL acrylic- and epoxy-based precursors. These studies can be further extended to 3D-patterning of other common responsive gels that are based on similar polymerization chemistry, thus tuning the responsivity of the system for various environments. This synthetic procedure, with its significant capabilities for extensions in structural as well as hydrogel variability, should prove enabling for the exploration of autonomous micro/nano-actuators both inspired by biological systems and those creatively engineered.
Keywords: gels · hybrid materials · multiphoton lithography · stimuliresponsive materials $\cdot$ actuation

[1] J. Monod, J.-P. Changeux, F. Jacob, J. Mol. Biol. 1963, 6, 306

[2] M. Ghyoot, C. D. Ridder, M. Jangoux, Zoomorphology 1987, 106, 279.

[3] R. M. Macnab, J. Bacteriol. 1999, 181, 7149.

[4] R. L. Sutherland, L. M. Mäthger, R. T. Hanlon, A. M. Urbas, M. O. Stone, J. Opt. Soc. Am 2008, 25, 588.

[5] M. A. C. Stuart, W. T. S. Huck, J. Genzer, M. Müller, C. Ober, M Stamm, G. B. SukhorukovI, I. Szleifer, V. V. Tsukruk, M. Urban, F. Winnik, S. Zauscher, I. Luzinov, S. Minko, Nat. Mater. 2010, 9, 101.

[6] H. Saito, Y. Takeoka, M. Watanabe, Chem. Comm. 2003, 2126.

[7] A. Sidorenko, T. Krupenkin, A. Taylor, P. Fratz, J. Aizenberg, Science 2007, 315, 487

[8] D. J. Beebe, J. S. Moore, J. M. Bauer, Qing Yu, R. H. Liu, C. Devadoss, B.-H. Jo, Nature 2000, 404, 588.

[9] G. P. Andreas Richter, Adv. Mater. 2009, 21, 979.

[10] H. G. Schild, Prog. Polym. Sci. 1992, 17, 163.

[11] X. Zhou, L. Weng, Q. Chen, J. Zhang, D. Shen, Z. Li, M. Shao, J. Xu, Polym. Int. 2003, 52, 1153.

[12] M. Irie, D. Kunwatchakun, Macromolecules 1986, 19, 2476.

[13] K. Kataoka, H. Miyazaki, M. Bunya, T. Okano, Y. Sakurai, J. Am. Chem. Soc. 1998, 120, 12694.

[14] B. Jeong, A. Gutowska, Trends Biotechnol. 2002, 20, 305.

[15] J. Ge, Y. Yin, Angew. Chem. Int. Ed. 2011, 50, 2.

[16] P. Kim, L. D. Zarzar, X. Zhao, A. Sidorenko, J. Aizenberg, Soft Matter 2010, 6, 750.

[17] L. D. Zarzar, P. Kim, J. Aizenberg, Adv. Mater. 2011, 23, 1442.

[18] S. J. Bryant, K. D. Hauch, B. D. Ratner, Macromolecules 2006, 39, 4395.

[19] T. Yu, C. K. Ober, Biomacromolecules 2003, 4, 1126.

[20] D. Y. Wong, D. R. Griffin, J. Reed, A. M. Kasko, Macromolecules 2010, 431, 2824.

[21] C. N. LaFratta, J. T. Fourkas, T. Baldacchini, R. A. Farrer, Angew. Chem. Int. Ed. 2007, 46, 6238.

[22] T. Baldacchini, C. N. LaFratta, R. A. Farrer, M. C. Teich, B. E. A Saleh, M. J. Naughton, J. T. Fourkasa), J. Appl. Phys. 2004, 95.

[23] P. J. Campagnola, D. M. Delguidice, G. A. Epling, K. D. Hoffacker, A. R. Howell, o. D. Pitts, S. L. Goodman, Macromolecules 2000, 33 1511.

[24] S. Li, L. Li, F. Wub, E. Wang, J. Photoch. Photobio. A 2009, 203, 211.

[25] S. J. Jhaveri, J. D. McMullen, R. Sijbesma, L.-S. Tan, W. Zipfel, C. K. Ober, Chem. Mater. 2009, 21, 2003.

[26] T. Watanabe, M. Akiyama, K. Totani, S. M. Kuebler, F. Stellacci, W. Wenseleers, K. Braun, S. R. Marder, J. W. Perry, Adv. Funct. Mater. 2002, 12, 611.

[27] B. Kaehr, J. B. Shear, P. Natl. Acad. Sci. USA 2008, 105, 8850.

[28] R. Nielson, B. Kaehr, J. B. Shear, Small 2009, 5, 120.

[29] S. K. De, N. R. Aluru, J. Microelectromech. S. 2002, 11, 544.

[30] B. Behkam, M. Sitti, Appl. Phys. Lett. 2007, 90, 023902.

[31] H. H. Motoi Oishi, Teppei Uno, Takehiko Ishii, Michihiro Iijima,, Y. Nagasaki, Macromol. Chem. Physic. 2007, 208, 1176.

[32] K. L. Heredia, Z. P. Tolstyka, H. D. Maynard, Macromolecules 2007, 40, 4772.

[33] D. C. Lin, B. Yurke, N. A. Langrana, J. Mater. Res. 2005, 20, 1456.

[34] K. Okeyoshi, R. Yoshida, Adv. Funct. Mater. 2010, 20, 708.

[35] K. Podual, F. J. D. III, N. A. Peppas, J. Control. Release 2000, 67, 9. 
Entry for the Table of Contents (Please choose one layout)

Layout 2:

\section{Writing Hydrogels on Pillars in 3D}

Lauren D. Zarzar, Philseok Kim, Mathias Kolle, C. Jeffrey Brinker, Joanna Aizenberg, * Bryan Kaehr*

$$
\text { Page - Page }
$$

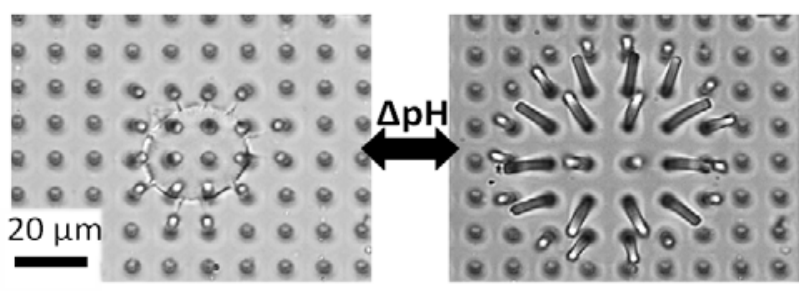

Direct Writing and Actuation of 3D-Patterned Hydrogel Pads on Micropillar Supports

We demonstrate that freely-swelling, three-dimensionally patterned responsive hydrogels fabricated with multiphoton lithography on the tips of flexible pillars provide unique capabilities for the design of adaptive systems with tunable actuation direction and angle, sensitive optical response, and precise spatial integration of gels of varying responsivity. 